\title{
EXCAVACIONES ARQUEOLÓGICAS EN Chazuta, San Martín. UN REPORTE DE CAMPO (PRIMERA TEMPORADA, 2002)
}

\section{GORI TUMI ECHEVARRÍA LÓPEZ'}

El presente documento es un reporte de la Primera Temporada de las labores de investigación arqueológica realizadas en el poblado de Chazuta, departamento de San Martín, en los andes amazónicos peruanos. Este trabajo se llevó a cabo en forma de rescate y se trazó como objetivo la excavación y recuperación de un conjunto de «urnas funerarias» encontradas dentro del área urbana del poblado y que corrían riesgo de ser destruidas por actos vandálicos locales.

El reporte se enfoca principalmente en la descripción de diez contextos de alfarería enterrada, la mayoría de ellas con probables fines funerarios. En este registro se destacan las cuadrículas 1 y 8 que presentaron contextos complejos, cuyo registro fue extensivo durante la intervención. Los resultados del trabajo exponen un conjunto de información que constituye por ahora el primer corpus de data arqueológica controlada obtenida de este poblado peruano.

The present document is a report of the First Season of archaeological investigations Andean carried out in the town of Chazuta, department of San Martin in the Amazon of Peru. This season was design to work like an salrage archaeologycal rescue and its principal objective was the excavation and the recovery of several «funerary urns» found within the urban area of the town that were in risk of being destroyed by local vandalic acts.

The report focuses mainly on the description of ten contexts of buried pottery, most of them with probable funeral functions. In this regis en the archaeological units 1 and 8 stand out displaying complex contexts whose registry was extensive during the intervention. The results of the work present an information set that so far constitutes the first corpus of controlled archaeological data from this Peruvian town.

$1 \quad$ Arqueólogo egresado de la Universidad Nacional Mayor de San Marcos, ha realizado investigaciones arqueológicas en Perú, México y Bolivia. Entre sus últimos trabajos destacan los estudios efectuados en el sitio de Choquequirao (Cusco) e investigaciones en los Llanos de Mojos en Bolivia. Actualmente es presidente de la Asociación Peruana de Arte Rupestre (APAR), asociada a la Federación Internacional de Organizaciones de Arte Rupestre (IFRAO), y es autor de variados artículos sobre su especialidad. 


\section{Introducción ${ }^{2}$}

El presente documento es un reporte resumido de los trabajos de excavación arqueológica llevados a cabo en el poblado de Chazuta, Departamento de San Martín, realizado el año 2002 por encargo del INC Departamental San Martín. Estos trabajos tuvieron el carácter de emergencia y se enfocaron en el rescate y documentación de un conjunto de «urnas funerarias» halladas dentro del perímetro urbano del poblado de Chazuta que estaban siendo vandalizadas y destruidas por los pobladores locales.

Estos trabajos estuvieron dirigidos por el arqueólogo Anselmo Lozano y fueron ejecutados en esta primera etapa por el que suscribe, quien se desempeñó como Jefe de Campo de las operaciones de excavación y registro de las evidencias arqueológicas. Todas las labores operativas de excavación, que fueron diseñadas bajo responsabilidad del Jefe de Campo, se realizaron entre el 5 y el 31 de octubre del 2002, y se centraron en el descubrimiento, excavación y rescate de «urnas funerarias» o materiales similares hallados a lo largo y ancho del poblado.

Este reporte, por la razón indicada, va a lidiar únicamente con los procedimientos y resultados de la intervención física en las zonas donde esta fue requerida, y no tratará sobre análisis materiales específicos u otro tipo de información relacionada. Al final del reporte no obstante se harán algunas conclusiones derivadas de la intervención y algunas apreciaciones sobre los resultados y sus implicancias culturales.

Debemos advertir finalmente que parte de la información vertida en este documento es incompleta debido fundamentalmente a la carencia documental del registro, el cual se encuentra en poder del arqueólogo Anselmo Lozano. Hasta el año 2007 solo parte de este material, dibujos de campo y fotografías del autor, fue devuelto al que suscribe, siendo usados para este reporte.

\section{Cuestiones previas}

\section{a) Antecedentes}

Aunque en Chazuta existen referencias locales, y algunos relatos referidos al hallazgo de vasijas desde tiempos históricos, las primeras noticias sistemáticas de la presencia de «urnas funerarias» en esta zona se deben al arqueólogo americano Warren DeBoer (1984), quien reportó haber reconocido «urnas» y material cerámico a nivel superficial y en cortes de caminos en varias secciones del poblado de Chazuta, considerando el componente arqueológico cerámico local como «extensivo».

\footnotetext{
2 Todos los gráficos, dibujos de plantas, cortes, perfiles y fotografías del presente artículo fueron realizados por Gori Tumi Echevarría López, excepto la Figura 3, que corresponde a Anselmo Lozano.
} 
De acuerdo a sus observaciones, DeBoer reconoció superficialmente una clara variación estilística en la realización de alfarería tradicional en Chazuta, la cual le permitió separar directamente dos estilos cerámicos, el «Old Chazuta style» referido al material arqueológico, y el «New Chazuta Style» referido al material contemporáneo. La distinción se basó únicamente en que el «Old Chazuta Style», carece casi por completo de los atributos estilísticos del complejo cerámico contemporáneo, lo cual puede ser cotejado etnográficamente hasta hoy.

Aunque DeBoer no confrontó su seriación con ninguna prueba estratigráfica, llegó a documentar literalmente la presencia de dos «urnas» funerarias en la Plaza de Armas del poblado, donde observó el afloramiento de las tapas cerámicas de estas urnas. DeBoer, salvo mejor información, no llegó a realizar excavaciones arqueológicas de ningún tipo, y se limitó a repetir descripciones particulares de la naturaleza física de estos contextos alfareros o «urnas funerarias».

Posteriormente, en diciembre del 2001, el arqueólogo Anselmo Lozano, del INC de San Martín, realiza una visita de inspección a solicitud de los vecinos de Chazuta en vista de la destrucción a que estaban siendo sometidas numerosas «urnas» enterradas en las inmediaciones del poblado. Lozano (comunicación personal) verifica in situ la existencia de un número variado de «urnas» dispersas en el poblado, la mayoría de ellas vandalizadas o rotas. De acuerdo al reporte de Lozano (Lozano, 2002) hasta la inspección quedaban seis «urnas funerarias» todavía intactas, y «en peligro de ser profanadas». La inspección solo duró un día y sirvió de base a la formulación del proyecto de rescate que se llevó a cabo, en su primera etapa, el año 2002.

\section{b) Ubicación geográfica}

Chazuta se encuentra ubicado en un recodo de la cuenca media baja del río Huallaga, sobre el banco aluvial de la quebrada del rió Chazuta, el mismo que es cortado por el cauce curvo del primer río en su margen izquierda (FIGURA 1). Aunque el territorio es todavía montañoso, Chazuta está ubicado en el límite de la selva alta, sobre el pie de monte proyectado al noreste, aproximadamente a 260 msnm. La base del poblado es una antigua terraza geológica sedimentada, donde predominan estratos de arcillas y arenas, al menos en la parte urbana del poblado.

Estando ubicado sobre la base de una cuenca semi encañonada, Chazuta es el primer asentamiento importante, en este decurso, entre la zona de selva alta y selva baja, habiendo sido históricamente un productor de cerámica y un puerto de intercambio comercial hasta mediados del siglo pasado (DeBoer, 1984). En la actualidad Chazuta es mejor caracterizado como un típico poblado andino amazónico. 


\section{c) Métodos y procedimientos}

Debido a que los trabajos se planificaron directamente sobre evidencia de alfarería enterrada, llamada simplemente «urnas», se usaron los siguientes parámetros metodológicos para una intervención de emergencia:

- $\quad$ El reconocimiento de las afloraciones físicas de la evidencia, mediante una limpieza superficial.

- El reconocimiento de la matriz mediante un pequeño test superficial con badilejo.

- La ubicación geodésica de las cuadrículas de excavación.

- Una excavación por niveles arbitrarios definidos de acuerdo al comportamiento geológico de los yacimientos.

- La excavación de una cuadrícula arbitraria orientada al norte, con posibilidades de ser ampliada, y con un retiro lo suficientemente ancho para que una persona pueda estar dentro de la cuadrícula.

- Dibujo de planta, de perfiles y cortes de los materiales excavados, según relevancia del registro. También se incluyó el fotografiado extensivo de la evidencia.

- La toma de alturas uniformes sujetas al levantamiento topográfico de toda el área de intervención.

- El retiro de todos los materiales hallados de acuerdo al número de cuadrícula, y el número de material correlativo.

- El depósito de todos los materiales en un almacén específico, en el Centro «Wasichay» de taller cerámico.

Adicionalmente, el que suscribe incluyó el concepto de «rasgo» para la identificación de hallazgos, sean éstos concentraciones de artefactos, o para las mismas urnas. Esta categoría se uso básicamente para la Cuadrícula 8 que mostró un contenido complejo, como se verá posteriormente.

Como es natural, algunos de los aspectos metodológicos de la intervención tuvieron que ser replanteados debido a factores de preservación de la evidencia, como por ejemplo la determinación de las matrices de las urnas enterradas, las que no fueron reconocidas en ninguno de los casos. Tampoco se siguió un parámetro de referencia para alturas porque, al menos hasta la participación del que suscribe, no se hicieron los levantamientos topográficos ofrecidos. 
Aunque todos los demás aspectos de la intervención fueron desarrollados normalmente, se presentaron problemas de conservación material debido a la mala estimación de los aspectos medioambientales hechos por el Director del Proyecto, quien determinó la apertura y exposición de todas las cuadrículas excavadas. Estas cuadrículas se abrieron en forma correlativa y se excavaron en dos grupos de manera simultánea ${ }^{3}$.

Esta forma de intervención, sin la extracción inmediata de los materiales excavados, generó problemas de conservación efectiva de todas las evidencias, especialmente debido al desecamiento de los materiales arcillosos del soporte de las vasijas que se habían dejado durante la excavación para sostener el material, y al mismo desecamiento de las vasijas, que sufrieron, casi en todos los casos, craquelamientos y fracturas. A esto se sumó, lamentablemente, la inundación de las cuadrículas $1,4,7,8,9$ y 10 por la lluvia del 22 de octubre.

\section{Resultados}

El que suscribe solo fue responsable de la intervención en 8 cuadrículas de excavación, aunque durante la intervención fueron excavadas dos cuadrículas adicionales, hechas por el Director del Proyecto con arqueólogos de la Universidad de Trujillo. Muchas de las cuadrículas que se abrieron no correspondieron a las «urnas» localizadas por el arqueólogo Anselmo Lozano durante su evaluación de diciembre del 2001, al menos éstas no fueron indicadas explícitamente, habiéndose reconocido nuevos sitios con materiales arqueológicos enterrados en toda el área de la población de Chazuta.

Aunque las cuadrículas abiertas correspondieron tanto a enterramiento de «urnas» como yacimientos arqueológicos convencionales (cerámica), todas las unidades se abrieron con el objetivo de localizar y «rescatar» urnas del tipo funerarias, que fue el objetivo primario de la intervención arqueológica. Todas las cuadrículas presentaron características particulares y se registraron en forma independiente por grupos de excavación, aunque los parámetros de intervención fueron uniformes para todos los casos, salvo las excavaciones particulares del Director de Proyecto.

El Mapa 2 (FIGURA 2) indica la ubicación referencial de los pozos o cuadrículas de excavación en el ámbito urbano del poblado de Chazuta. Aunque este plano muestra claramente las relaciones espaciales de las unidades de excavación, no se debe dejar de anotar que absolutamente todas las cuadrículas se encuentran dentro del área urbanizada del poblado. Las cuadrículas siguieron una numeración

Uno de los grupos estuvo compuesto por el Director del Proyecto y dos licenciados en arqueología de la Universidad Nacional de Trujillo. El otro grupo estuvo formado por el Jefe de Campo y obreros locales. 
correlativa y prosiguieron casi de acuerdo a las posibilidades de intervención singular, muchas veces dentro de casas particulares, o al aire libre.

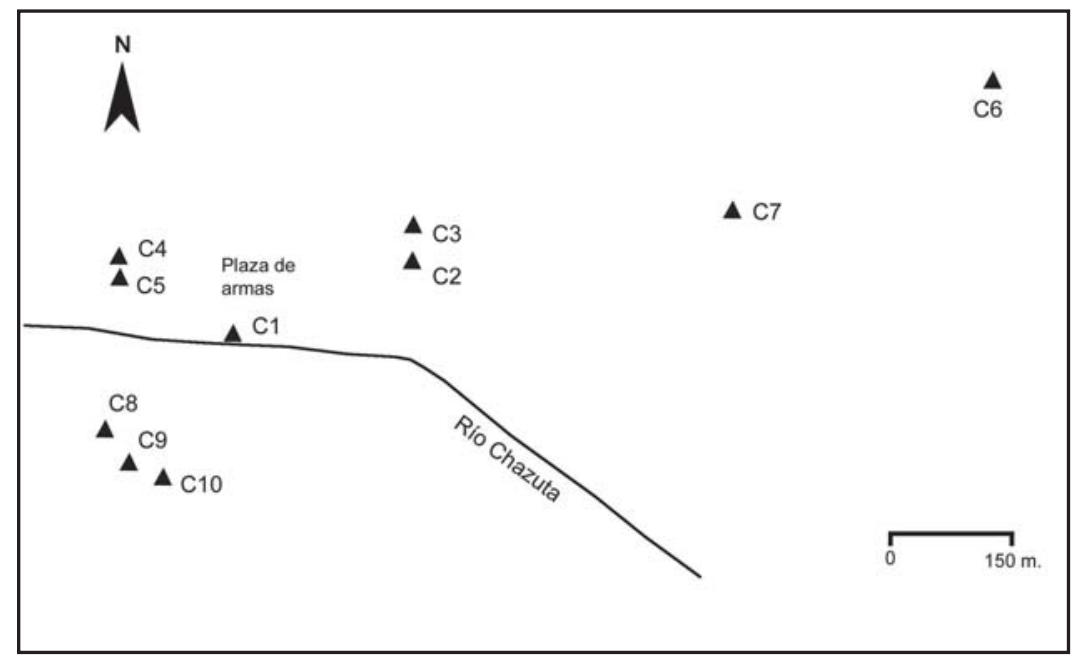

Figura 2.

Mapa de distribución de cuadrículas excavadas en el ámbito urbano de Chazuta

En general, sólo 4 cuadrículas presentaron material en situación original, el resto se mostró vandalizado o destruido, y este porcentaje puede ser incluso menor siguiendo una proyección negativa respecto a la conservación de otras vasijas enterradas en el poblado; no obstante esto, la mayoría de estos contextos presentaron un vandalismo localizado en la parte superior, o en la «tapa» de la evidencia, la que se presenta generalmente rota al momento del hallazgo; es bastante probable dada la profundidad relativa de enterramiento de algunas «urnas» o vasijas, que en la mayoría de estos contextos, al menos la mitad inferior de la unidad compuesta (tapa y base) se encuentre en buen estado de conservación.

Hasta aquí la falta de los registros originales nos limita respecto a una exposición de resultados con más detalles, especialmente dada la división de los grupos para excavaciones simultáneas; así tenemos que los arqueólogos trujillanos excavaron y registraron las cuadrículas 2, 5, 6 y 9; y bajo control del que suscribe se excavaron las cuadrículas 1, 3, 4, 7 y 8 , con resultados positivos para vasijas o urnas sólo en las cuadrículas 1 y 8 . Aunque se hallaron materiales arqueológicos en las cuadrículas 2, 5, 6 y 9, todos estos registros permanecen en poder del arqueólogo Lozano, y no se conocen en detalle las particularidades de los mismos.

Aunque dada la evidencia es claro que solo podemos exponer con cierta holgura los resultados de la excavación en la Cuadrículas 1 y 8 , vamos a tratar de 
describir muy parcialmente las demás cuadrículas para un mejor acercamiento a la naturaleza de la evidencia arqueológica reconocida en Chazuta.

Cuadrícula 2 (C2). Se trató de un contexto intacto; una vasija globular o tinaja de pasta crema y una especie de cuenco como cubierta, de aproximadamente $45 \mathrm{cms}$ de alto. La pieza que hacía las veces de tapa presentaba un cuerpo cónico y una base plana por lo que se colige que la abertura de la boca de la vasija de base debió ser muy reducida. Es probable que esta no haya servido como contenedor funerario. No se presentaron asociaciones de ningún tipo y tampoco se pudo reconocer la matriz de la vasija.

Cuadrícula 3 (C3). Una excavación que se hizo bajo la presunción de la presencia de una urna, después de dos días de excavaciones no se halló ningún indicio arqueológico y se abandonó la unidad.

Cuadrícula 4 (C4). Se excavó una vasija totalmente destruída pero compactada como un bloque sólido. Esta se halló sobre una de las calles del poblado. Es posible que esta vasija halla sido destruida por vandalismo y luego arrojada sobre su mismo pozo para luego ser fuertemente apisonada por el paso de los autos.

Cuadrícula 5 (C5). Una cuadrícula abierta unos metros al sur de la unidad anterior, sólo se recuperaron algunas piezas cerámicas. Este pozo se cerró por que no produjo mayor información.

Cuadrícula 6 (C6). Una inmensa y verdadera urna, de casi $1.5 \mathrm{~m}$ de alto y un peso aproximado de más de 100 kilos. Esta tremenda pieza de alfarería fue ubicada en el segundo pozo de cateo dentro de una casa al este del poblado. De acuerdo a las personas de la casa esta vasija se halló cuando se hicieron trabajos de remodelación interno. La vasija no obstante fue vandalizada pues presentó un fuerte orificio por rotura en su sección superior o «tapa» por el que se filtró abundante tierra. Debido al peso de la vasija se procedió a retirar parte de la tierra del interior, para su levantamiento respectivo.

Lamentablemente esta vasija atravesó un proceso de remoción muy estresante por lo que su parte superior sufrió numerosas fracturas. Esta urna tuvo que ser trasladada en camioneta hasta el depósito designado donde probablemente permanece hasta hoy.

Cuadrícula 7 (C7). Una excavación realizada en una de las colinas de la falta norte que limita el poblado. Aquí se observó la impronta de vasijas por lo que se procedió ha hacer una pequeña cala hasta los $20 \mathrm{cms}$. de profundidad recuperándose abundante material cerámico de variadas tipologías en el tratamiento de pasta, restos óseos y algunos artefactos, como un piruro. Esta unidad lamentablemente no arrojó evidencias de alguna vasija enterrada, interpretándose el contexto como el despojo 
de una excavación antigua en los alrededores, donde se ha documentado otros probables contextos de vasijas enterradas.

Cuadrícula 9 (C9). Una cuadrícula excavada al pie de una casa hacia la banda sur del poblado, margen derecha del río Chazuta. Aquí se pudo documentar una vasija enterrada con algunas ofrendas de vasijas cerámicas menores asociadas.

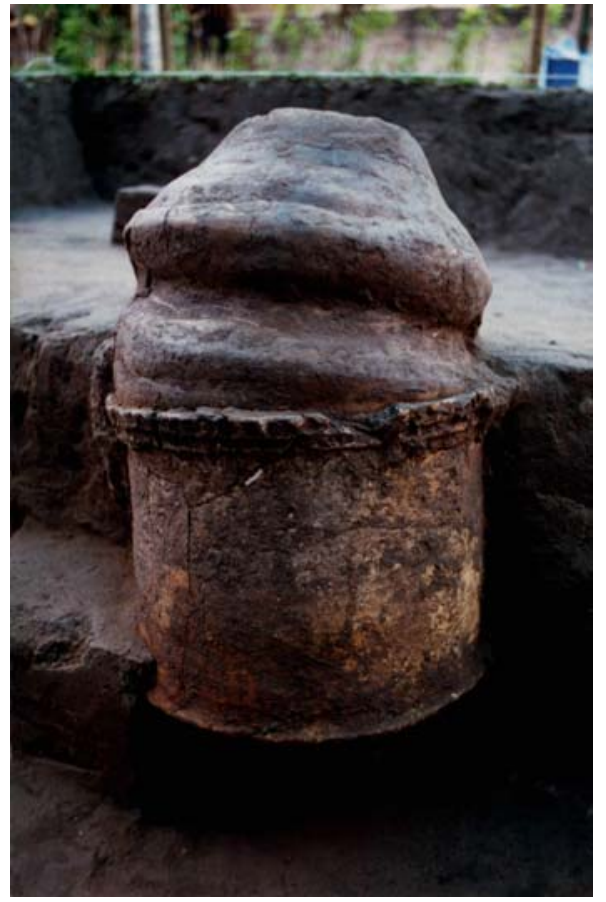

Figura 3.

C10, urna de cuerpo tubular
Cuadrícula 10 (C10). Una urna tubular sobre el camino, cerca de la unidad anterior. Esta pieza presentó un sorprendente buen estado de conservación y una variación formal notable, especialmente para su base, mostrando un cuerpo tubular con líneas moldeadas en relieve en los vértices superiores e inferiores de su volumen principal (FIGURA 3), constituyendo un ejemplo interesante de la variación formal de las vasijas enterradas en Chazuta. Esta vasija fue excavada por el arqueólogo Anselmo Lozano.

Las dos restantes unidades, que constituyen el cuerpo de la evidencia de este reporte, presentan al menos dibujos de planta, cortes y perfiles, además de una selección muy concisa de fotografías, no obstante esta presentación debemos advertir que es posible complementar estos datos con nueva información, especialmente gráfica, de los registros originales.

\section{Cuadrícula 1 (C1)}

Se trató de una unidad diseñada para intervenir el afloramiento de la parte superior de una supuesta «urna» (FIGURA 4), ésta aparecía en superficie como una impronta circular en el piso irregular de una cocina común dentro de una casa, en la vereda este de la plaza de armas de Chazuta. Esta área fue nivelada anteriormente y mediante este proceso se habían levantado y destruido 2 vasijas enterradas adicionales, ubicadas a menos de 3 metros de la primera, de las cuales solo quedaban algunas improntas muy deterioradas. Este dato resultó muy interesante porque fue el primer indicio de un patrón de ubicación concentrada de cerámica enterrada en el poblado. 
La unidad se excavó mediante tres niveles arbitrarios. Aunque superficialmente se pudo reconocer básicamente el contenido del primer nivel (0-2 cms), este presentó una cobertura de desechos modernos (plástico, cerámica, basura orgánica y piedras pequeñas) sobre una tierra húmeda predominantemente arenosa con poca arcilla, la cual estaba siendo apelmazada por el constante trajín de las personas al interior de la casa.

El $2^{\circ}$ nivel excavado bajó hasta los $30 \mathrm{cms}$, atravesándose la capa de basura superficial que rodeaba a la parte superior de la vasija. Este nivel consistió de varias capas de desechos acumulados y superpuestos, incluyendo lentes de ceniza, desechos orgánicos y basura moderna. Todo este material se interpretó como producto de la acumulación de desechos y la remoción superficial de tierra y los restos aflorantes de la vasija enterrada. Hacia la esquina noreste incluso se pudo reconocer un pozo de desechos con material orgánico y basura moderna.

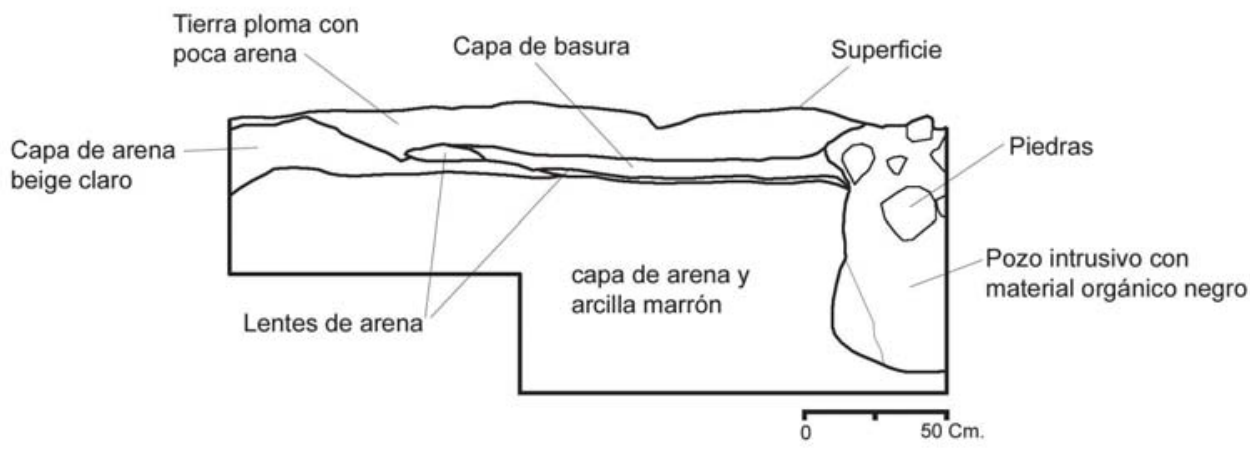

Figura 4.

C1, corte superficial con los restos de la vasija aflorante.

A partir del 3er nivel, bajo los 30 cms., el terreno consistió de una capa de tierra de un color marrón compuesta por arena y arcilla, ligeramente con más contenido de este último material; esta capa se presentó menos húmeda que la superficie y con una consistencia más suave (FIGURA 5). Esta es la capa que cubrió originalmente la vasija. La vasija presentó en superficie un diámetro de $53 \mathrm{cms}$, y un espesor de la pasta de $1.5 \mathrm{cms}$, cuando se alcanzó la base de la excavación, aproximadamente a los $70 \mathrm{cms}$, desde la superficie del perfil sur, la vasija, una urna, mostró una morfología compuesta, base y tapa, con un cuerpo globular, tipo olla, el cual había sido cubierto por otra pieza de otra morfología, pues presentaba la boca más abierta y las paredes exteriores del cuerpo en forma recta, aunque lamentablemente con la base destruida (FIGURAS 6, 7 y 8). 


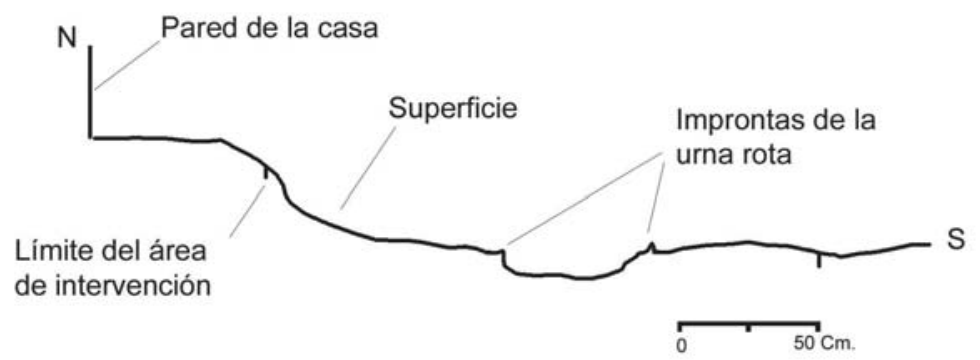

Figura 5.

C1, Perfil Norte.

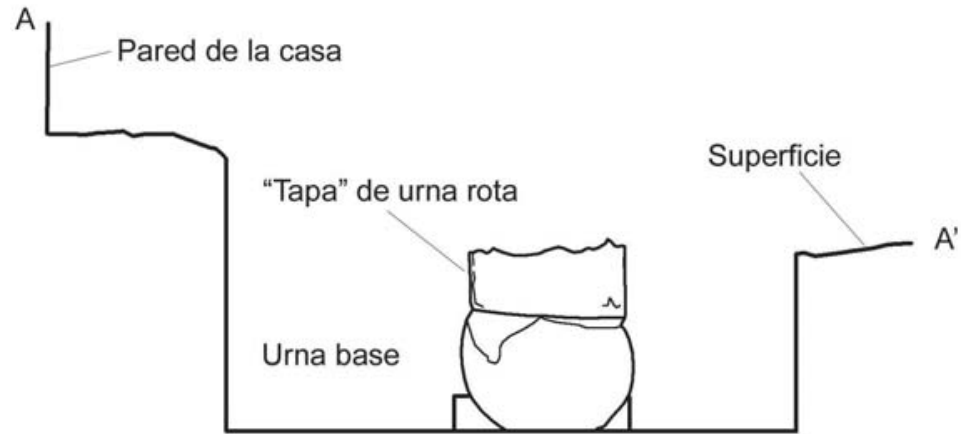

Figura 6.

C1, planta de la unidad excavada.
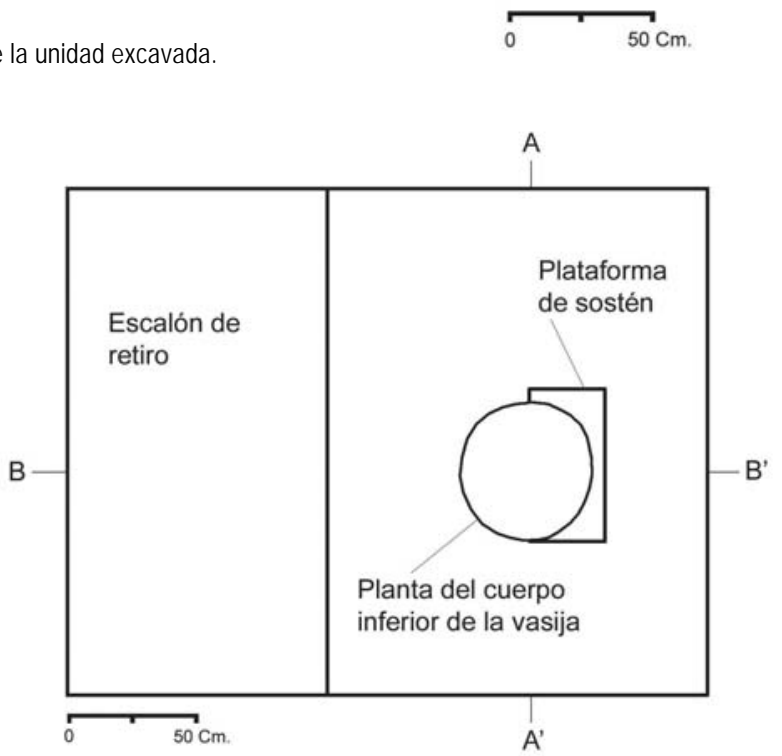

Figura 7.

C1, corte A-A' mostrando la urna excavada. 


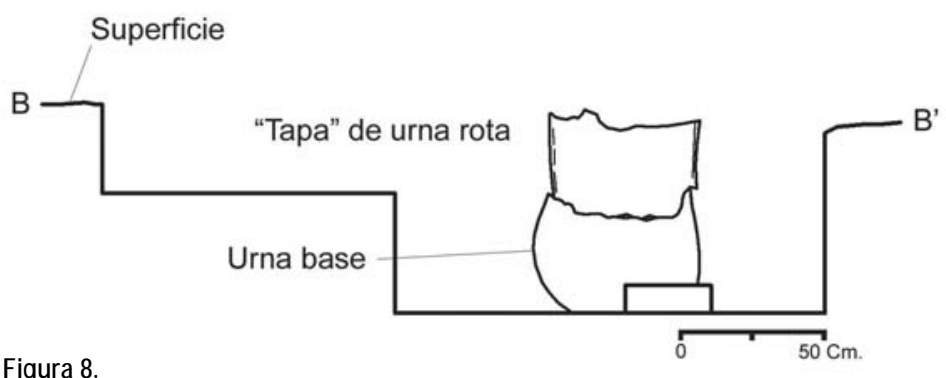

Figura 8.

C1, corte B-B' mostrando la urna excavada.

La comprobación de la naturaleza compuesta de esta evidencia constituyó la primera prueba real de la presencia de urnas en la zona; por otro lado la identificación de la variación formal en la articulación de la composición de este contexto cerámico, porque no se ha comprobado aún que hubiera restos humanos en estas vasijas, significó también evidencia interesante y primaria de un patrón regular en el enterramiento arqueológico de estos materiales. Aunque esta urna presentó un evidente vandalismo o destrucción en su cima (FIGURA 9) fue el ejemplo más relevante de este tipo de material obtenido dentro del poblado.

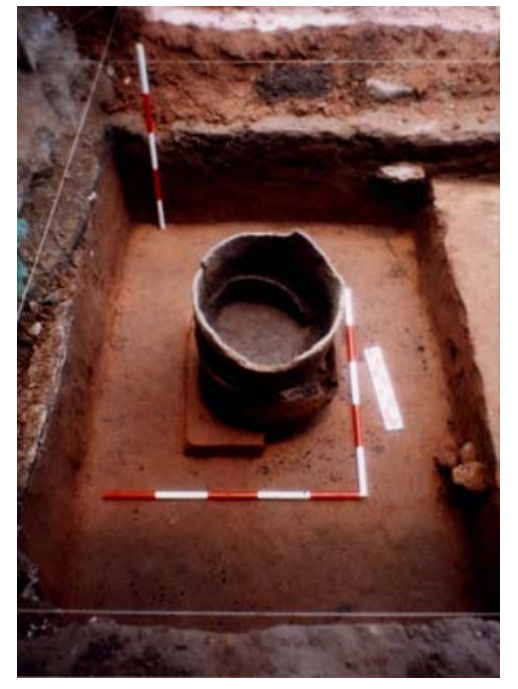

Figura 9.

C1, vista desde arriba de la urna excavada.

\section{Cuadrícula 8 (C8)}

Esta cuadrícula representó también una de las excavaciones más interesantes de toda la intervención. Esta se llevó a cabo en el patio de una escuela hacia la margen derecha del río Chazuta en una zona que es conocida como «la banda» del poblado (ver FIGURA 2). La cuadrícula se trazó sobre una zona expuesta de gras ralo y tierra 
donde se supone fue localizada una «urna» por algunos miembros de la escuela. En esta área se delineó una cuadrícula de $2 \times 2 \mathrm{~m}$ y se procedió a excavar un nivel arbitrario de $40 \mathrm{cms}$. Hasta casi alcanzar el límite de este nivel, pegado al perfil norte de la cuadrícula, se pudo hallar los bordes superficiales de la vasija (Rasgo $\mathrm{N}^{\circ}$ 1) que se estaba tratando de localizar (FIGURAS 10 y 11).

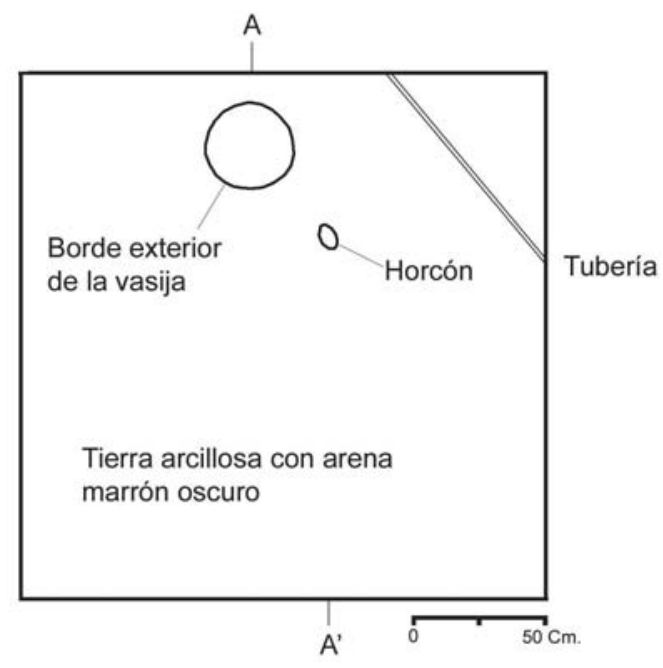

Figura 10.

C8, planta final del 1er nivel de excavación mostrando los bordes expuestos de la vasija.

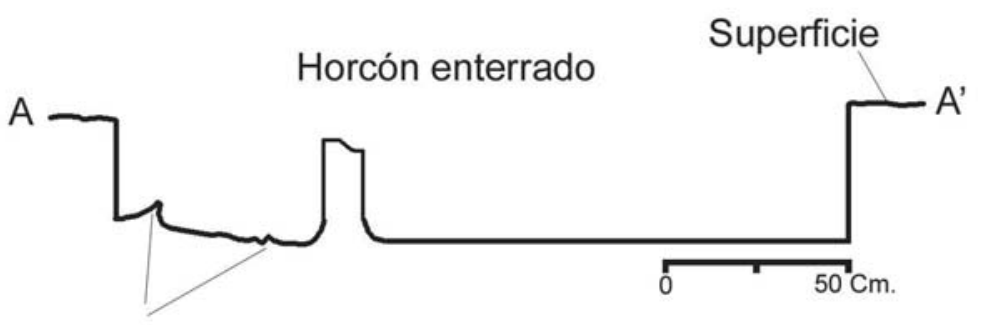

Bordes exteriores

de la vasija

Figura 11.

C8, corte A-A' de los bordes expuestos de la vasija. 
Hasta los $20 \mathrm{cms}$, se pudo registrar basura moderna, lentes de ceniza y depósitos de tierra disturbados por actividades extractivas, como la excavación de pequeños pozos para postes, de los cuales se localizó un horcón todavía in situ. Los $20 \mathrm{cms}$ restantes del 1er nivel mostraron una regularidad material y un contexto inalterado de deposición de suelo salvo algunas zonas. El 2do nivel excavado expuso la misma composición continuada de material original, aunque no se pudo reconocer el cuerpo de la vasija debido a su forma cónica. Hay que destacar que algunas secciones de la cuadrícula sufrieron procesos disturbativos variados, y es lo que probablemente perjudicó la cima de la vasija hallada o Rasgo $\mathrm{N}^{\circ} 1$, la cual no presentó cubierta o tapa, que debió perderse por vandalismo.

La capa inferior consistió de arcilla y arena limpia de color marrón claro, esta consistencia es característica del suelo geológico del sitio que sirvió de sustrato prácticamente a todas las deposiciones de vasijas o urnas en la zona. En este caso cubrió prácticamente todo el cuerpo inferior del Rasgo $\mathrm{N}^{\mathrm{o}} 1$, y hasta su localización todo el Rasgo $\mathrm{N}^{\circ}$ 2, que fue hallado exactamente en la esquina noroeste de la cuadrícula original excavada (FIGURAS 12 y 13). El Rasgo $\mathrm{N}^{o} 2$, se trató de otra vasija, esta vez intacta, y motivó la apertura de una cuadrícula de expansión hacia el noroeste de la unidad original. Hasta aquí no se presentó evidencia que estos dos rasgos estuvieran asociados de alguna manera; salvo la clara relación espacial, no existen materiales arqueológicos asociados de ningún tipo o algún contexto vinculante, como domésticos u otros.

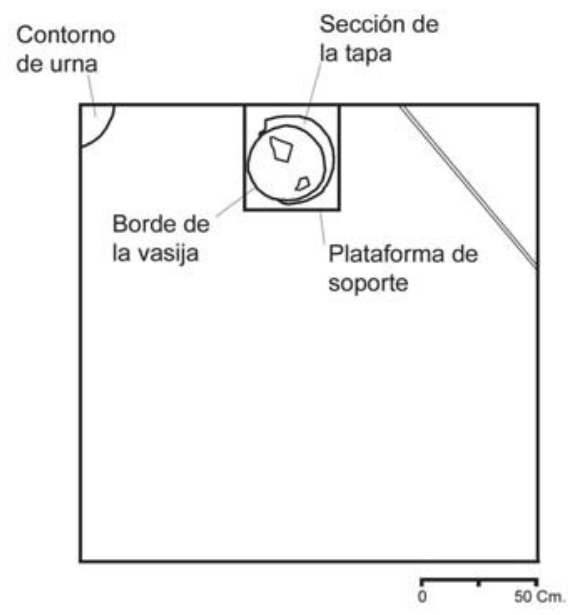

Figura 12.

C8, planta de la unidad mostrando el Rasgo No 1, y el contorno del Rasgo No 2

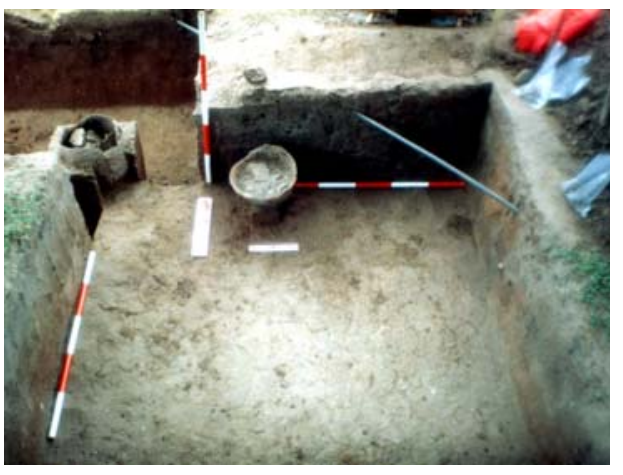

Figura 13.

C8, vista de la unidad mostrando los Rasgos Nos 1 y 2. 
La apertura de la nueva cuadrícula siguió los mismos parámetros metodológicos de la unidad original y se expandió, desde un retiro de $50 \mathrm{cms}$., un metro por lado conformando un cuadrante regular de 1.5 por lado. Toda el área se excavó a su vez en dos niveles dejando la vasija sobre una plataforma cuadrangular cubriendo dos tercios inferiores de su base y dejando expuesta solo su cima descubierta, y lo mismo se hizo con el Rasgo $\mathrm{N}^{\mathrm{o}} 1$ cuya plataforma de sostén cubrió el cuerpo de la vasija. Cuando se completaba el segundo nivel de esta excavación se descubrió un tercer rasgo ubicado en el borde del perfil oeste de la nueva cuadrícula; este rasgo consistió de una tercer contexto cerámico: una «tapa» y una vasija asociada (Figura 14).

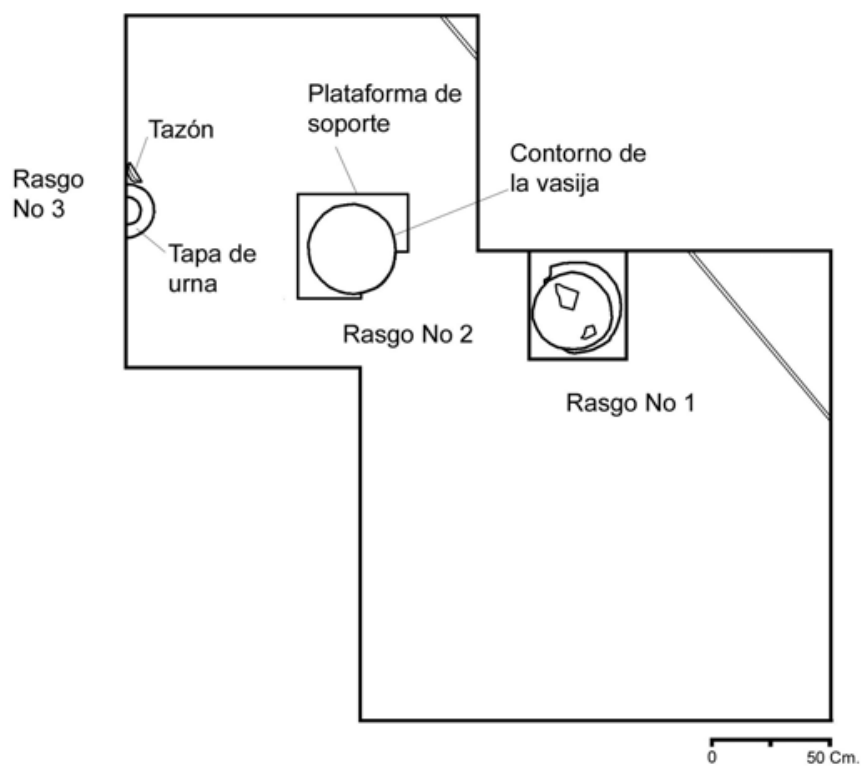

Figura 14.

C8, Planta compuesta de la unidad, mostrando los Rasgos Nos 1, 2 y 3.

Este hallazgo obligó a ejecutar una segunda cuadrícula de ampliación, la que se excavó siguiendo los mismos condicionamientos anteriores, solo se alteraron la profundidad en el último nivel en $30 \mathrm{cms}$. adicionales, para las dos áreas adicionales, dejando una sección escalonada en referencia a la cuadrícula original. Esto se hizo para permitir una mejor intervención en los nuevos descubrimientos. El comportamiento estratigráfico fue el mismo que en la unidad original salvo variaciones en la capa superior moderna que no son relevantes de destacar. Esta última intervención arrojó adicionalmente dos nuevos rasgos: Rasgo $\mathrm{N}^{\circ} 4$, consistente en un conjunto de horcones y piedras; y Rasgo $\mathrm{N}^{0}$ 5, una vasija aislada (Figura 15). 


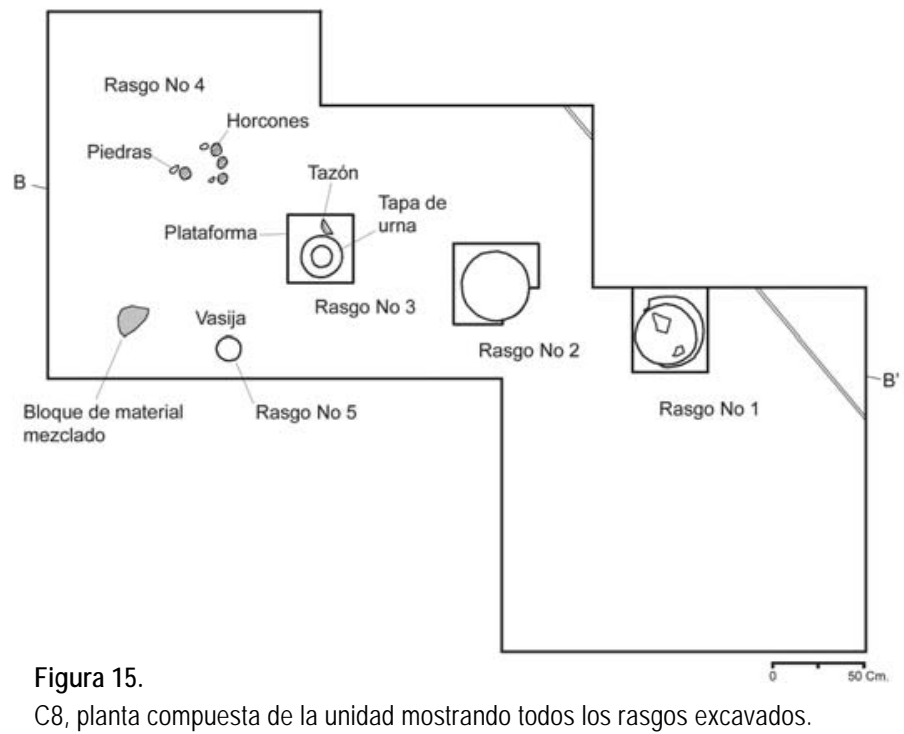

Posteriormente se comprobó que los horcones y las piedras constituyeron un contexto intrusivo moderno, lo cual se interpretó como consecuencia del uso tardío de la pampa abierta en el patio de la escuela, lo que es coincidente con el horcón hallado en la cuadrícula original. Los demás hallazgos constituyeron contextos cerámicos originales no explícitamente relacionados por algún medio físico, o alguna evidencia vinculante. Hacia la esquina sur oeste de esta última cuadrícula se halló además un bloque de desechos con carbones, arcillas y tierra mezclados lo que podría sugerir quizá restos de alguna actividad doméstica relacionada, pero también apareció en forma aislada como todos los rasgos descubiertos.

Hasta finalizar la excavación de esta cuadrícula, era claro que los rasgos se presentaron en forma independiente, muy probablemente como resultado de procesos de deposición particulares (figura 16), todos los contextos cerámicos mostraron una composición dual con piezas variadas en los cuatro casos en que se presentaron estas muestras Rasgos $\mathrm{N}^{\circ} 1,2,3$ y 5 ; aunque los Rasgos $\mathrm{N}^{\circ} 1$ y $\mathrm{N}^{\circ} 5$ presentaron únicamente piezas singulares. El Rasgo 1 consistió de una vasija de forma cónica con base circular (figura 17), mientras el Rasgo 2 no pudo ser formalmente identificado salvo la parte superior que mostró una vasija invertida (tapa), fracturada por cusas naturales, pero de evidente contorno circular (Ver figura 13).

Es probable que los Rasgos $\mathrm{N}^{0} 3$ y $\mathrm{N}^{0} 5$ estén asociados, aunque esto merece ser todavía comprobado estableciendo más bases comparativas con más investigación; no obstante, el enterramiento de una vasija en forma aislada (Rasgo $\mathrm{N}^{\circ} 5$, ver figura 15) causa suspicacias si se toma en cuenta que el Rasgo $\mathrm{N}^{\circ} 3$ presenta 
otra vasija similar (tazón) inmediatamente asociada al conjunto, y la C9 presentó dos piezas similares juntas. En cualquier caso el hecho que el Rasgo $\mathrm{N}^{\circ} 5$ se encuentre disociado de contextos más complejos es un indicador relevante de la alta variación deposicional hallada en este sitio.

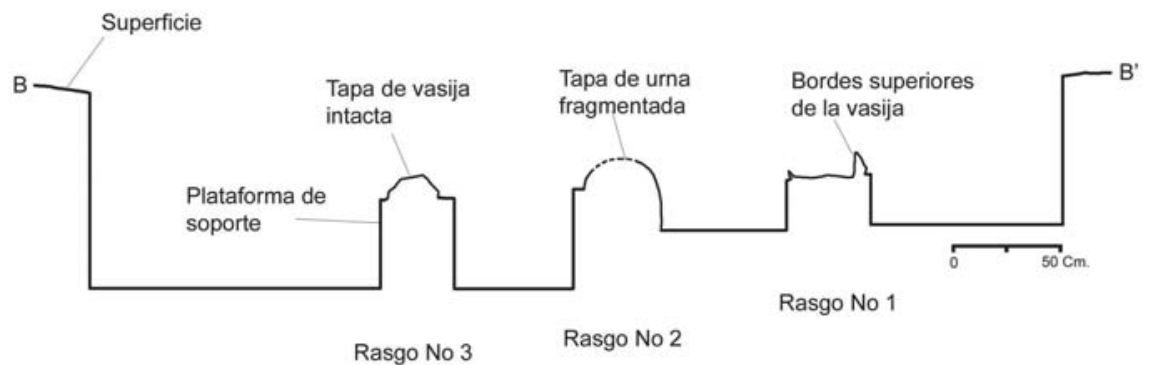

rigura 10.

C8, corte B-B' de la unidad mostrando los principales rasgos excavados.

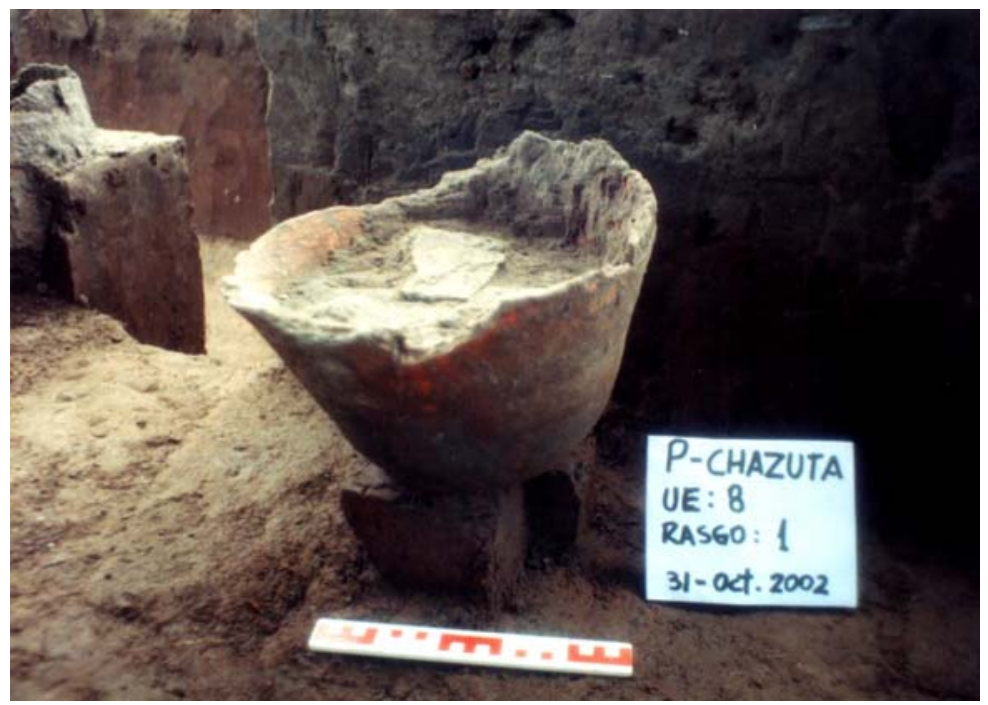

\section{Figura 17.}

C8, Rasgo No 1

Hay que indicar el Rasgo $\mathrm{N}^{\circ} 3$ consiste de dos vasijas superpuestas, una base y su tapa, y una pequeña vasija colocada en forma vertical inmediatamente al costado y en la parte media de las dos vasijas superpuestas. La vasija inferior del par tiene una forma cilíndrica corta de paredes rectas aunque algo evertidas hacia la base y los bordes, asemejando más a una fuente que a una olla. La vasija superior, por otro lado, presentó la forma de una olla carenada de perfil compuesto, claramente con la 
abertura de la boca de diámetro inferior al de la vasija de base, lo cual es un hecho interesante; la vasija más pequeña asociada es claramente un tazón (figura 18).

Hasta aquí es claro que existe una alta variación formal respecto al uso de vasijas para enterramientos, es evidente que los conjuntos son siempre compuestos y no incluyen, en ninguno de los casos observados repeticiones morfológicas destacables, salvo la presencia de los tazones asociados. Esto contrasta con el evidente patrón de ubicación concentrada que ya había sido advertido en la Cuadrícula 1, y que se ha repetido en esta última cuadrícula. La variación formal puede sugerir cierta independencia significativa entre los materiales enterrados, aunque no estamos en capacidad de establecer aún cuales son las implicancias culturales de estas variaciones.

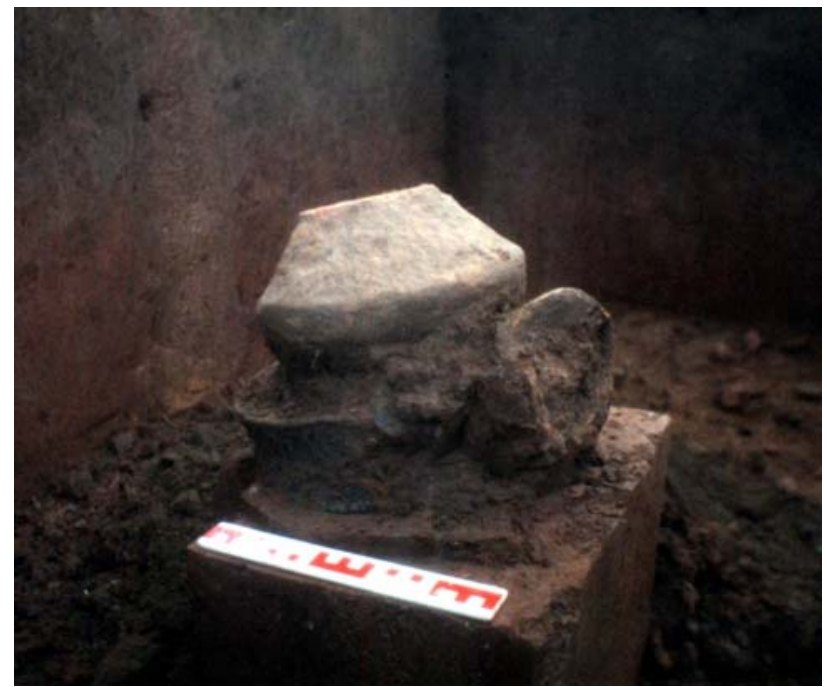

Figura 18.

C8, Rasgo No 3.

\section{Conclusiones}

Dada la cantidad de información recuperada y expuesta no se pueden establecer extensas relaciones de valor cultural salvo algunas conclusiones relacionadas, en este sentido en primer lugar debemos resaltar el notable valor arqueológico de Chazuta como continente de una extensa variación de contextos cerámicos enterrados, los cuales son comúnmente denominados «urnas funerarias». La cantidad de estos entierros materiales debió ser muy extensa a juzgar por el gran número de restos de cerámica presentes en los alrededores del poblado actual. Conforme se extiende la población, los entierros cerámicos van apareciendo y se vienen vandalizando o destruyendo, lo que constituye una verdadera desgracia. 
Aunque la intervención de rescate ha logrado documentar una muestra muy pequeña de este tipo de contextos arqueológicos, es evidente, en relación a la distribución de los materiales excavados, que la zona donde se ubica el poblado actual de Chazuta sirvió de soporte a una actividad relacionada al enterramiento de contextos cerámicos complejos, probablemente de uso funerario. A pesar que nosotros no reconocimos explícitamente este uso, y hay que mencionarlo, al menos hasta el final de mi intervención, esta es una tradición muy común en toda la Amazonía y constituye probablemente parte de la variación funcional a la que estuvieron sujetos estos enterramientos. Es evidente que sugerimos más de un uso relacionado al enterramiento de vasijas en Chazuta.

No creo que Chazuta sea un área exclusivamente de enterramiento de vasijas, en varias de las cuadrículas se pudo reconocer también fragmentería cerámica asociada de variada topología en el tratamiento de pasta, además de áreas con carbones, huesos, arcillas en pasta, etc., lo que sugiere la existencia de un complejo arqueológico mayor, del cual estas vasijas enterradas son sólo un remanente. DeBoer (1984) también advirtió la presencia de depósitos con fragmentería cerámica relacionada al «Old Chazuta Style» lo que implica un componente adicional al panorama arqueológico del área. Sobre esta información es casi concluyente que el sitio actual de Chazuta debió ser territorio de un asentamiento complejo antiguo (arqueológico) del cual quedan dispersadas las vasijas enterradas.

Debemos destacar nuevamente que las vasijas enterradas mostraron un claro patrón de concentración como se pudo corroborar en las C1, C8 y en los alrededores de la C7 que no reportó enterramiento alguno. Lamentablemente no se conocen los límites específicos de estas acumulaciones, si llegan a formar superposiciones, o si se intruyen. El hecho que las vasijas enterradas tengan una gran dispersión solo indica primariamente un área de cobertura pero no un patrón específico de disposición o acumulación de vasijas. Las concentraciones pueden indicar clusters de unidades con relevancias culturales dentro del desarrollo interno del asentamiento arqueológico.

Otros elementos interesantes son la notable variación de las vasijas enterradas tanto a nivel formal como en las dimensiones. $\mathrm{C} 1$ expuso claramente una urna con forma de olla como base, y una vasija de cuerpo cilíndrico de tapa; $\mathrm{C} 2$ por su parte mostró una tinaja cuya tapa fue básicamente una especie de cuenco de lados evertidos. De otro lado C6 mostró otra urna u olla grande, la más grande de la muestra; y C10 expuso una vasija cilíndrica de cuerpo alargado recto con una tapa de vasija de perfil compuesto carenado, esta última vasija muy similar al Rasgo $\mathrm{N}^{\circ}$ 3 de $\mathrm{C} 8$.

Sobre esta variación no obstante existe una regularidad en el tratamiento de las vasijas, predominando lo que podríamos llamar un «estilo llano», sin decoraciones pictóricas o patrones escultóricos. Aunque la pasta exterior es llana, es probable que 
exista un tratamiento mínimo que aún no ha sido ampliamente advertido, como el engobado de algunas vasijas ( $\mathrm{C} 1$ y $\mathrm{C} 10)$, y un patrón corrugado en algunas otras piezas cerámicas expuestas (Rasgo $\mathrm{N}^{\circ} 2, \mathrm{C} 8$ ). Excepto la urna de C10, que presentó una moldura decorativa con impresión digital en forma de banda en el remate superior del cilindro (Ver FIGURA 3), ninguna otra vasija de los contextos examinados presentó decoración adicional alguna. De este grupo deben excluirse no obstante los pequeños tazones asociados a estos contextos que presentaron una decoración geométrica por incisión cortante en una banda sobre el tercio superior exterior de la vasija.

Dentro de la muestra, estas variables son altamente representativas, pero esperan aún ser ajustadas para establecer hipótesis sobre sus implicancias culturales, lo que se debe a la limitación del registro ahora disponible, y a la falta de análisis extensivos. La colección formada por esta primera temporada fue bastante interesante, y creo que eso es algo evidente a estas alturas; estamos seguros que sobre este parámetro todo el nuevo material recuperado por la segunda campaña debió ser igualmente impresionante. Espero que la pronta incorporación de nueva información proveniente de los registros originales, y de las nuevas temporadas, complementarán este reporte en bien de la historia de Chazuta y de la arqueología nacional. 
Bibliografía

\section{ECHEVARRÍA LÓPEZ, Gori Tumi}

2002 Informe No 001-220-GEL-PCH_SM. Informe de actividades del mes de octubre del 2002. Chazuta, San Martín.

2002 Diario de Campo. Proyecto de Evaluación y Rescate. Urnas Funerarias de Chazuta, Departamento de San Martín. Manuscrito.

\section{DEBOER, Warren}

1984 Archaeological Reconnaissance in the Central Huallaga, Department of San Martin. Northeastern Peru. Flushing, N.Y. Queens College, Laboratory of Anthropology. USA.

\section{LOZANO CALDERÓN, Anselmo}

2002 Proyecto de Evaluación y Rescate Arqueológico de Urnas Funerarias. Chazuta, Departamento de San Martín. Moyabamba. 